AperTO - Archivio Istituzionale Open Access dell'Università di Torino

\title{
Personalized Spatial Support for People with Autism Spectrum Disorder
}

\section{This is the author's manuscript}

Original Citation:

Availability:

This version is available http://hdl.handle.net/2318/1690970

since 2019-02-07T14:26:42Z

Publisher:

ACM

Published version:

DOI:10.1145/3213586.3225229

Terms of use:

Open Access

Anyone can freely access the full text of works made available as "Open Access". Works made available under a Creative Commons license can be used according to the terms and conditions of said license. Use of all other works requires consent of the right holder (author or publisher) if not exempted from copyright protection by the applicable law. 


\section{Personalized Spatial Support for People with Autism Spectrum Disorder}

\author{
Federica Cena \\ Department of Computer Science \\ University of Torino \\ Italy \\ federica.cena@unito.it
}

\author{
Amon Rapp \\ Department of Computer Science \\ University of Torino \\ Italy \\ amon.rapp@gmail.com
}

\author{
Claudio Mattutino \\ Department of Computer Science \\ University of Torino \\ Italy \\ claudio.mattutino@unito.it
}

\begin{abstract}
This paper presents a novel personalized service to support people with Autism Spectrum Disorder (ASD) in their daily movements within urban environments. It aims at helping them in managing anxiety and stress originated by routine breakdowns and unfamiliar situations, improving their autonomy. In particular, we provide an interactive map that is i) personalized, i.e., able to recommend "Safe" Point-of-Interests (SPOIs) according to users' preferences for and aversions to places' sensory features; ii) crowdsourced, i.e., populated with comments and reviews by people with ASD and their caregivers; iii) assistive, i.e., able to support everyday movements (homework, home-relatives, etc.) by presenting "safe" paths to reach desired places. In this paper we will focus on the first aspect of the map, i.e. SPOIs recommendations. The presented approach is part of a wider project, PIUMA, Personalized Interactive Urban Maps for Autism, which has the aim to develop novel digital solutions for helping people with cognitive problems in their everyday movements.
\end{abstract}

\section{KEYWORDS}

Adaptive support, recommender systems, assistive technologies, Personalized maps, autism

\section{ACM Reference Format:}

Federica Cena, Amon Rapp, and Claudio Mattutino. 2018. Personalized Spatial Support for People with Autism Spectrum Disorder. In UMAP'18 Adjunct: 26th Conference on User Modeling, Adaptation and Personalization Adjunct, July 8-11, 2018, Singapore, Singapore. ACM, NY, NY, USA, 6 pages. https://doi.org/10.1145/3213586.3226218

Permission to make digital or hard copies of all or part of this work for personal or classroom use is granted without fee provided that copies are not made or distributed for profit or commercial advantage and that copies bear this notice and the full citation on the first page. Copyrights for components of this work owned by others than the author(s) must be honored. Abstracting with credit is permitted. To copy otherwise, or republish, to post on servers or to redistribute to lists, requires prior specific permission and/or a fee. Request permissions from Permissions@acm.org.

UMAP'18 Adjunct, July 8-11, 2018, Singapore, Singapore

(c) 2018 Copyright is held by the owner/author(s). Publication rights licensed to ACM. ACM ISBN 978-1-4503-5784-5/18/07...\$15.00

https://doi.org/10.1145/3213586.3225229

\section{INTRODUCTION}

ASD is considered an increasing health problem, with 1 in 68 children affected by ASD in United States today [17]. ASD people show problems in social interaction, communication, attention, and practical skills [13]. In particular, ASD is marked by an atypical social functioning, with a tendency to or even a need of withdrawal from social interactions. The consequences may range from a preference for non-socially intensive jobs to actual seclusion. ASD is also characterized by weak central coherence, that is a reduced capacity for extracting a Gestalt from a collection of details [12]. In short, these persons tend to see the trees rather than the forest. Moreover, people with ASD often react abnormally to sensory experiences. Most of them are particularly and even painfully sensitive to certain sounds, lights, tastes and smells, being overwhelmed by sensory inputs that are easily managed by neurotypical ${ }^{1}$ individuals. For ASD individuals, the brain seems unable to balance the senses appropriately [26].

At least in part because of these characteristics, persons with ASD tend to have a reduced range of activities and interests, often preferring mechanical, deterministic situations, having the need to find reassurance by sticking to rigid, repetitious routines. This turns into problems in managing unexpected events. ASD persons are often affected by high levels of anxiety that they hardly cope with, thus preventing them to accomplish their daily activities [29]. All these characteristics may imply peculiarity in spatial representation and the need of personalized supports for orientation and movements in urban environments. To better understand their "spatial needs", we preliminary interviewed twelve individuals (six in a first phase [24], and other six in a second phase) with ASD and invited them to draw some cognitive maps. On the one hand, interviews were aimed to explore ASD people everyday "spatial routines", their urban landmarks, their use of digital map services, and their requests of support. On the other hand, cognitive maps, which are mental representations of specific spaces, were used to identify ASD

\footnotetext{
${ }^{1}$ A word used in the ASD community to identify people who are not on the autism spectrum.
} 
individuals' representation modalities of city environments, as well as to understand how they commonly move during a typical day. Cognitive maps were collected by eliciting participants to sketch their city center and the neighborhood where they lived.

Results highlighted that ASD individuals have fixed routines in terms of daily movements, whereby breakdowns lead to anxiety and the incapability of managing the unexpected event. Moreover, they expressed a need to receive support on how to socially behave in specific situations. Other requirements were related to the willingness of visiting places that present "safe" characteristics, such as being scarcely crowded, or with smooth lights and quiet. Features of such places mostly depended on individual preferences, since sensory sensitivity seems highly idiosyncratic.

The project described in this paper, PIUMA (Personalized Interactive Urban Maps for Autism), aims to give a technologyenhanced orientation support to adolescent/adult individuals with medium (who may present moderate-impaired cognitive ability), or high functioning autism (who present normal mental functioning), or with Asperger's Syndrome (now categorized as a form of ASD in the DSM-52). In particular, the project aims at designing and developing tools to practically support people with ASD in their daily movements within urban environments, helping them manage anxiety and stress originated by routine breakdowns and unfamiliar situations, as well as improving their autonomy. We provide an interactive map that is i) personalized, i.e., able to recommend "Safe" Point-of-Interests (SPOIs) according to users' preferences for and aversions to places' sensory characteristics; ii) crowdsourced, i.e., populated with comments and reviews by people with ASD and their caregivers; iii) assistive, i.e., able to support them in their everyday movements (home-work, home-relatives, etc.) by presenting "safe" paths to reach their desired places.

The rest of the paper will focus on the first aspect, i.e., SPOIs recommendations, and it is structured as follows: related work is presented in Section 2, while Section 3 describes a scenario illustrating an example of application. Section 4 outlines our recommendation approach. Finally, we conclude and propose future work in Section 5.

\section{RELATED WORK}

Computer-based interventions appear to be particularly promising for supporting ASD people, since their particular affinity to technology [23]. Different research has recently used technology to address specific problems of ASD, such as social communications [1], emotion recognition [11], learning [32], and collaboration for design $[2,8]$. However, such works mainly focused on ASD children [3, 7], leaving apart autistic adults. Second, they mostly preferred to address social behavior problems [25]. Third, they treated ASD deficits in artificial environments, making difficult the transfer of improvements gained during the treatment to the real world [11, 31]. What we

\footnotetext{
${ }^{2}$ Diagnostic and Statistical Manual of Mental Disorders, Fifth Edition
}

propose, instead, is specifically addressed to ASD adults, aiming at supporting them more in their everyday transfers and tasks, than in their social relations, through interactive tools that are fully integrated in their daily environments.

Moreover, it is worth to say that ASD can manifest itself in different forms, where limitations can range from people with multiple impairments to high-functioning autistic people, with no cognitive deficits [24]: even within comparable levels of skills, the ways ASD affects behavioral patterns, perceptual sensitivities, special interests vary widely making one-size-fitsall technology not viable [13]. Nevertheless, personalized technologies for autism are still very rare [29].

Most of the applications investigating the adoption of personalization strategies for people affected by autism regard the educational domain [19], e.g., [15], a personalized e-learning system that exploits semantic web technologies, and [20], an adaptive Web-based application to help autistic students overcome the challenges they may face when going to university. The idea of providing people affected by autism with recommendations and suggestions is preliminarily investigated by Hong et al. [14], who proposed a social network aiming at supporting the independence of young adults with autism. Differently from this work, which is mainly based on crowdbased advice, our approach further exploits personal preferences and other characteristics of the person to better drive the personalization and support process. The effectiveness of personalization strategies in this domain has been recently shown by Khosla et al. who reported in [16] the results of a ninemonth trial investigating the impact of an adaptive humanoid robot able to change its behavior (voice tone, expressions, sound effects) on the ground of the activity patterns and the preferences of the participants.

Regarding spatial support for disabilities, to our knowledge, none of the works available in literature provides personalized spatial support for cognitive impairments. Previous approaches focus on other kinds of, mostly physical, disabilities (limited mobility, blindness, etc.). Typically, crowdsourced maps allow users to review the physical accessibility of specific POIs for wheelchair users, such as Wheelmap $\left.{ }^{3}[5,6]\right)$. These georeferenced crowdsourced data are also used to develop routing algorithms for people with mobility impairment, e.g., in RouteCheckr [32] and mPass [22]. Finally, there is a lot of research in the field of POIs and route recommendation, considering both user and context model [9, 10, 18, 27, 30], but no applications focuses on recommending POIs and routes that are perceived as safe by people with specific cognitive disabilities.

To summarize, PIUMA present four novel aspects: i) it addresses adult people with ASD who are commonly overlooked by current research in favor of children; ii) it supports them also in tasks different from social communication and learning, like movements and urban transfers; iii) it goes beyond the limitations of behavioral interventions conducted in artificial

\footnotetext{
${ }^{3}$ http://wheelmap.org/en/
} 
environments, by designing tools to be used in everyday situations; iv) it provides personalized tools adapted to the ASD peculiarities as well as to specific user and context features.

\section{SCENARIO}

Tom is 27 years old and lives with her mother. He suffers from high-functioning ASD. He has a high-school diploma in computer science and works as technical assistant in a big technology store. He likes videogame and computers, but avoids "offline" social interactions. He uses a wearable device to record his steps, heart rate and other physiological parameters (such as skin conductance). Tom commonly goes to work alone by bus. $\mathrm{He}$ is very methodical and routine-lover, and he performs more or less the same actions, as well as travels the same paths, every day. When something goes wrong (for example, his bus is canceled), he usually becomes stressed and anxious, and he does not know what to do. In this case PIUMA might provide him with the right type of support, in terms of content and modality, considering his preferences (he likes videogames and computers), aversions (he does not like crowded or too bright places), "internal states" (stress), context (time and weather). For example, if the routine breakdown happens in the morning, when traffic is very heavy, and Tom's stress level is getting higher (detected by his wearable bracelet), the system can directly call his mother to receive help (A). In another case, if the breakdown happens after work, when the weather is nice, the traffic is lighter, and Tom's stress level is lower, it might suggest a place where to relax and find calm. In this case, it can recommend to reach a videogame shop in the nearby area, on the basis of his interests in videogames and computers, since at that time the shop is not very crowded and has smooth lights, being the right place where to stay for him (B). After he enjoyed the place, if the bus line problem has still not been solved, the system may suggest an alternative route to go back home by foot, following isolated streets next to a park (C).

\section{PERSONALIZED SPATIAL SUPPORT}

The main goal of PIUMA project is to assist users' routine, supporting everyday movements of ASD people (home-relatives, home-work, etc.) by providing tailored helps for facing breakdowns from routines. In our perspective, such support should be personalized not only according to the user's preferences and interests, but also taking into account her peculiar problems with sensorial modalities, in order to provide comfortable "safe" POIs, as less annoying as possible. Moreover, personalized support could be provided according to the user's current level of stress and anxiety.

For example, as seen in the scenario above, if the bus line the user commonly takes is canceled for any reason, the system will provide her with suggestions about how to reach the target place (e.g. suggesting an alternative "safe" path), or recommendations about alternative "safe" places to be reached, or a communication channel with a caregiver who can provide help. Such spatial support is personalized on the basis of the user's preferences (what she likes and dislikes), habits and current emotional status (when available, thanks to wearable sensors, such as a wristband that detects the level of arousal or stress). Thus, the typology of support (calling a caregiver (A), recommending novel safe places (B) or suggesting an alternative path to reach the target one (C)) is decided on the fly according to the particular user's status (e.g., if she is very stressed, a human support could be better). The content of the support will be personalized according to the user and her context as well, e.g., the suggested POIs and alternative path are tailored to the user's preferences, for example by recommending a less crowded route if she does not like other people, and adapted to the specific moment of the request. Let's see more in details how the recommendations process works.

\subsection{Safe POIs and safe paths}

As said, in case of a breakdown of routine, which may yield a state of stress, the system can suggest an alternative "safe" path to reach the target place, or an alternate "safe" POI to be reached, according to the user's preferences.

A safe POI is defined as a reference point location identifiable and/or reachable in case of user's reorientation is in need. Each node on the map may be a candidate to represent a safe POI, but, to be safe, the POI should have the following characteristics: be familiar for the subject, comfortable (e.g., not too noisy, or not too crowded, according to the idiosyncratic user's sensory dispositions), and easy to reach (visible and well placed: e.g., no crossings needed, clear and accessible entrance from the street, etc.).

The features that a place needs to have to be defined as "safe" for a person with ASD have been defined by a team of two psychologists, one neuropsychiatry, and two experts in humancomputer interaction after twenty interviews to ASD individuals. Interviews lasted about one hour each and were audio recorded. They aimed at identifying ASD people's preferences about outdoor and indoor places, with reference to e.g. the degree of social interaction required, the number of people present, the sensory experience elicited. Interviews were transcribed verbatim and analyzed through a thematic analysis. The findings were coded separately by two researchers. Then, the researchers reviewed together the generated codes and resolved inconsistencies. The resulting codes were finally grouped into themes. By and large, interviews on the one hand confirmed literature results: ASD people rarely perceive as comfortable places that may cause high sensory stimulation [26]. On the other hand, they revealed that each ASD person has her own aversions, and there are no places' features that may reassure the whole ASD population. For example, P4 recounted how she is not bothered at all by the visual aspects of a place, such as strong lights, rather being negatively affected by loud sounds originated from people chatting each other, or rumors coming from trafficked streets. By contrast, P7 said that she is indifferent to crowds, actually she avoids too silent places, but that the narrowness of a place dramatically impacts on her sense of safety and comfort. In sum, the safe nature of a place is a matter of personal dispositions and idiosyncratic preferences thus requiring personalized interventions. 
For this reason, in the registration phase to the system, we ask users to select aspects that they bear less in a place, chosen among those that emerged as most stressful and uncomfortable during the interviews: quite/noisy, isolated/crowded, cold/warm, narrow/big, bright/dark. For example, a user, by rating these aspects through a score that spans from 0 to 4 , can express that she has a strong aversion to bright (4), cold (3), and crowded (4) places. This information, together with her preferences for categories of POIs, and the context data, will contribute to determine our recommendations results (Section 4).

Safe POIs could include both places that the user already knows and appreciated in the past, and new places suggested by the recommendation engine, based on crowdsourced information provided by other people through the interaction with PIUMA map (Section 3.2).

The same rationale is followed for the definition of "safe" paths, i.e., paths that satisfy the perceptual needs of ASD people. So, for example, for those that are inclined to avoid noisy places, trafficked routes are not selected, as well as routes near schools, hospitals, or shopping areas, marketplaces, road works. By contrast, more quiet routes for pedestrians, with benches and trees, far from the most beaten streets, and next to parks, are chosen. To have this information, we use tags and annotations in OpenStreetMap ${ }^{4}$, as well as open data made available by the Municipality of Torino. However, a detailed description of this part of the project goes beyond the scope of this paper.

\subsection{Crowdsourced data about SPOIs}

Open data made available by web sites like OpenStreetMap, which provides tags and comments about places, are often incomplete, or, when present, they often do not contain the specific sensorial information we need for the project. For this reason, the PIUMA map exploits crowdsourcing mechanisms ${ }^{5}$, i.e. it is populated with POIs, comments, reviews, trails both by people with ASD and their caregivers, as well as by anyone wishing to contribute to the improvement of ASD people's lives (both in their city of residence and in other locations, which could be visited for tourism or work). To this aim, we designed an interface that appears in overlay on the map, where the user can rate (from 0 to 4 , using a slider) a place with reference to the following five features: i) level of noise; ii) level of crowding, iii) temperature, iv) level of brightness, v) spaciousness. Moreover, a global evaluation about the "comfort" of the place can be provided. Such information will create the domain knowledge base to be used as a source for the personalized recommendations provided by the system.

The implementation of the crowdmapping in PIUMA is based on FirstLife ${ }^{6}$, a social network based on interactive maps (see fig.1). The interactive map is based on AngularJS, Ionic, Leaflet and OpenStreetMap. FirstLife is a flexible platform that can be adapted to different aims. Its architecture is composed of an

\footnotetext{
${ }^{4}$ https://www.openstreetmap.org

${ }^{5}$ http:// www.maps4all.firstlife.org

${ }^{6} \mathrm{http}: / / \mathrm{www}$. firstlife.org
}

interactive geographical map-based interface as a frontend and a backend aimed at managing and searching geographical data. It allows to insert and manage different kinds of POIs directly from the map, and supports crowdsourced data collection. The platform also provides social networking features such as the user's profile and activity stream, a dashboard with notifications and messaging.

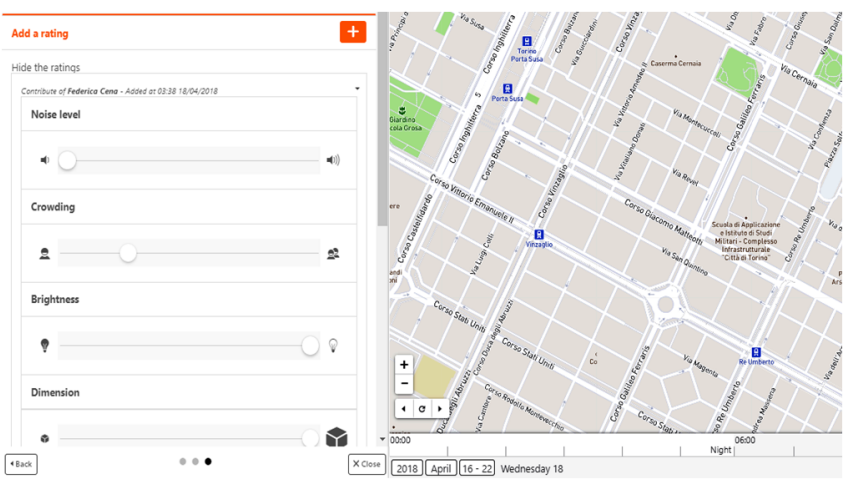

Figure 1. Screenshot of the crowdsourced map to insert evaluation on POIs' characteristics

\section{RECOMMENDATION ALGORITHM}

In order to order to generate POIs recommendations, a hybrid recommender in form of "cascade" approach has been implemented [4]. The "cascade" recommendation is performed as a sequential process where each recommender refines the recommendations given by the previous one. We start with a content-based recommender [21] and then we filter its results exploiting a collaborative-filtering recommender [28].

\subsection{Content-based}

First of all, a content-based recommender is implemented in order to filter the data based on the user's preferences over POI's categories. At the first interaction, we ask the user to rate from 0 to 4 some photos representing important categories of "urban daily living” (e.g., restaurants, pubs, shops, parks, libraries, ..), extracted from OpenStreetMap. We select only a subset of such categories (the most used in OpenStreetMap) in order not to overload the user.

Thus, for each of the preferred categories, we select the items that i) the user has already visited and rated positively, or that ii) are in the nearby (into a circle of 500 meters) and reachable by means of a "safe" routing. Then, among such places, we select POIs with sensory characteristics that match the user's preferences (or, better, that do not bother her too much). In doing so, we used the crowdsourced data collected through the interactive crowdsourced map, considering the particular time of the request and the time of the review (e.g., a place can be rated as crowded but only in particular moments of the day). 


\subsection{Collaborative filtering}

Afterwards, we implemented a collaborative filtering user-touser approach for a more fine-grained selection. In traditional collaborative filtering process, the similarity among users only depends on having rated similarly the same items. For the particular characteristics of ASD people, the things that they do not like are more important than the things that they like, for the severe consequence on stress they may have. Thus, we enrich the notion of similarity among two users to include similar aversions. As a consequence, in the similarity calculation, we considered not only the overall rating ascribed to the POIs, but also the similar users' aversions. For example, in the computation of similarity, two users that both hate bright and cold places are considered more similar than other two that do not share the same hassles.

So, we exploit Pearson correlations to find similarity among users and then we make a classic prediction considering the POIs that similar users (i.e., individuals who share the same aversions of the user) liked more (rated 3 o 4).

\subsection{Preliminary Evaluation}

When fully working, the system will be populated by assessments that users will make of the various POIs present in their city, using the interface for crowdsourcing. Since this part of the system is not yet fully active, in order to verify the correct behavior of the recommendation algorithm, the database has been filled by an automatic generator of users, with their ratings from 0 to 4 on the POIs' features as well as on the POIs' categories. The evaluations of the POIs that should come from the crowdsourced map are currently randomly simulated as well.

In the test the prediction accuracy is calculated using MAE and RMSE metrics. The calculation of the accuracy is made on $20 \%$ of the total number of POIs (test set) and several iterations with different test sets have been made. Both the MAE and RMSE are quite good (MAE: 0.04 and RMSE: 0.12), but we are aware that we need to test the algorithm with real ratings of people.

\section{FUTURE WORK AND CONCLUSION}

PIUMA is an ongoing project and many activities are still in progress. We cite here the most relevant ones for the conference aims.

i) Evaluation with real users: we plan to evaluate the accuracy of the recommendations with real users' ratings and profiles.

ii) Automatic stress/anxiety detection: we are working on detection of the level of stress/anxiety by using ad hoc wearable wristband, and by automatically detecting anomalies in the user's movements, exploiting the smart phone's sensors (e.g., identifying wandering, or prolonged staying in the same place, which may indicate confusion and loss of orientation)

ii) Adaptation of the map interface: the graphical presentation of the map will consider both the typical ASD deficits and the idiosyncratic user's cognitive disabilities (which may be relevant for individuals affected by mid-functioning autism), for example avoiding some unnecessary details not to burden or distract the user, or changing the interface modality according to her context and needs (text, audio, graphical, etc.).

iii) Routine breakdown detection: we are working on a mechanism for learning users' "spatial habits" and interruptions of such routines. Machine learning techniques for activity and plan recognition will be employed to this aim.

iv) Learning users' aversions: instead of asking users which POIs' features they do not like, we plan to learn such features by analyzing the users' ratings of POIs in the crowdsourced map, and their overall evaluation of the POIs, as well as considering the free text inserted as comments.

v) Learning users' preferences for POIs: instead of asking users to explicit their preferences rating photos in the registration phase, we will learn them by exploiting Facebook Graph API7, which allows developers to access information about check-ins and likes about places.

PIUMA aims at making cities accessible to people with cognitive disabilities, going beyond the mere physical accessibility addressed by most of the commercial map-based services currently available. It can be adapted to other cognitive disabilities, such as persons with Alzheimer's disease, after effects of head injury, and spatial agnosia.

\section{ACKNOWLEDGMENTS}

This work is supported by the COMPAGNIA di SAN PAOLO foundation, Member of the European Foundation Centre. Thanks to Marta Morante and Stefania Brighenti for their contribution to the work.

\section{REFERENCES}

[1] LouAnne E. Boyd, Alejandro Rangel, Helen Tomimbang, Andrea ConejoToledo, Kanika Patel, Monica Tentori, and Gillian R. Hayes. 2016. SayWAT: Augmenting Face-to-Face Conversations for Adults with Autism. In Proceedings of the $2016 \mathrm{CHI}$ Conference on Human Factors in Computing Systems (CHI '16). ACM, New York, NY, USA, 4872-4883.

[2] Benoît Bossavit and Sarah Parsons. 2016. "This is how I want to learn": High Functioning Autistic Teens Co-Designing a Serious Game. In Proceedings of the 2016 CHI Conference on Human Factors in Computing Systems (CHI '16). ACM, New York, NY, USA, 1294-1299. DOI: https://doi.org/10.1145/2858036.2858322

[3] Sofiane Boucenna, Antonio Narzisi, Elodie Tilmont, Filippo Muratori, Giovanni Pioggia, David Cohen, Mohamed Chetouani. 2014 Interactive Technologies for Autistic Children: A Review. Cognitive Computation, 6, 722 740

[4] R. D. Burke. 2002. Hybrid recommender systems: Survey and experiments. In: User Modeling and User-Adapted Interaction, Springer.

[5] Carlos Cardonha, Diego Gallo, Priscilla Avegliano, Ricardo Herrmann, Fernando Koch, and Sergio Borger. 2013. A crowdsourcing platform for the construction of accessibility maps. In Proceedings of the 10th International Cross-Disciplinary Conference on Web Accessibility (W4A '13). ACM, New York, NY, USA, , Article 26, 4 pages.

DOI=http://dx.doi.org/10.1145/2461121.2461129

[6] Sara Comai, S., Kayange, D., Mangiarotti, R., Matteucci, M., Ugur Yavuz, S. \& Valentini, F., Mapping city accessibility: review and analysis. Studies Health Technology and Informatics, 217, pp. 325-331, 2015

[7] Geraldine Dawson, and Karen Burner. 2011. Behavioral interventions in children and adolescents with autism spectrum disorder: a review of recent findings. Current opinion in pediatrics, 23(6), 616-620.

[8] Christopher Frauenberger, Julia Makhaeva, and Katharina Spiel. 2016. Designing Smart Objects with Autistic Children: Four Design Exposès. In Proceedings of the $2016 \mathrm{CHI}$ Conference on Human Factors in Computing

\footnotetext{
${ }^{7} \mathrm{https}: / /$ developers.facebook.com/docs/graph-api
} 
Systems (CHI '16). ACM, New York, NY, USA, 130-139.

[9] Ander Garcia, Olatz Arbelaitz, Maria Teresa Linaza, Pieter Vansteenwegen, and Wouter Sou.riau. 2010. Personalized Tourist Route Generation. In Proceedings of the 10th International Conference on Current Trends inWeb Engineering (ICWE'10). Springer-Verlag, Berlin,

[10] Damianos Gavalas, Michael Kenteris, Charalampos Konstantopoulos, and Grammati Pantziou. 2012. Web application for recommending personalised mobile tourist routes. Software, IET 6, 4 (2012), 313-322.

[11] Ofer Golan, Emma Ashwin, Yael Granader, Suzy McClintock, Kate Day, Victoria Leggett,Simon Baron-Cohen .2010. Enhancing emotion recognition in children with autism spectrum conditions: an intervention using animated vehicles with real emotional faces. Journal of autism and developmental disorders 40, 3 (2010), 269-279.

[12] Francesca Happé, Uta Frith. 2006. The weak coherence account: detail-focused cognitive style in autism spectrum disorders. J. of Autism and Developmental Disorders, 36(1): 5-25.

[13] R. Peter Hobson. 1995. Autism and the development of mind. Psychology Press. 10

[14] Hong, Hwajung, et al. 2012. Designing a social network to support the independence of young adults with autism. ACM CSWC12

[15] M. V. Judy, U. Krishnakumar and Hari Narayanan. 2012. Constructing a personalized e-learning system for students with autism based on soft semantic web technologies. 2012 IEEE International Conference on Technology Enhanced Education (ICTEE), Kerala, 2012, pp. 1-5. doi 10.1109/ICTEE.2012.6208625

[16] Rajiv Khosla, Khanh Nguyen and Mei-Tai Chu. 2015. Service personalisation of assistive robot for autism care. IECON 2015 - 41st Annual Conference of the IEEE Industrial Electronics Society, Yokohama, 2015, pp. 002088-002093. doi: 10.1109/IECON.2015.7392409

[17] Leigh J. P., Du J. 2015. Brief Report: Forecasting the Economic Burden of Autism in 2015 and 2025 in the United States. J Autism Dev Disord. 2015 Dec; 45(12), 4135-4139

[18] Kwan Hui Lim, Jeffrey Chan, Christopher Leckie, and Shanika Karunasekera. 2015. Personalized tour recommendation based on user interests and points of interest visit durations. In Proceedings of the 24th International Conference on Artificial Intelligence (IJCAI'15), Qiang Yang and Michael Wooldridge (Eds.). AAAI Press 1778-1784.

[19] Joseph Mintz and Morten Aagaard . 2012. The application of persuasive technology to educational settings. Educational Technology Research and Development 60(3) 483-499

[20] Alejandro Montes García, Natalia Stash, Marc Fabri, Paul De Bra, George H. L. Fletcher, Mykola Pechenizkiy. 2016. Adaptive web-based educational application for autistic students. Published in: Extended Proceedings of Hypertext 2016, Halifax, Canada. http://ceur-ws.org/Vol-1628/Demo1.pdf

[21] Michael J. Pazzani - Daniel Billsus. 2007 Content-Based Recommendation Systems, Springer.

[22] Catia Prandi, Paola Salomoni, Silvia Mirri. 2014. mPASS: integrating people sensing and crowdsourcing to map urban accessibility. 2014 IEEE 11th Consumer Communications and Networking Conference (CCNC), Las Vegas, NV, 2014, pp. 591-595.

[23] Sathiyaprakash Ramdoss, Wendy Machalicek, Mandy Rispoli, Austin Mulloy,
Russell Lang, and Mark O'Reilly. 2012. Computer-based interventions to improve social and emotional skills in individuals with autism spectrum disorders: A systematic review. Developmental Neurorehabilitation 15, 2 (2012), 119-135. DOI:http: //dx.doi.org/10.3109/17518423.2011.651655

[24] Amon Rapp, Federica Cena, Guido Boella, Alessio Antonini, Alessia Calafiore Stefania Buccoliero, Maurizio Tirassa, Roberto Keller, Romina Castaldo, and Stefania Brighenti. 2017. Interactive Urban Maps for People with Autism Spectrum Disorder. In Proceedings of the 2017 CHI Conference Extended Abstracts on Human Factors in Computing Systems (CHI EA '17). ACM, New York, NY, USA, 1987-1992. DOI: https://doi.org/10.1145/3027063.3053145

[25] Kathryn E. Ringland, Christine T. Wolf, Heather Faucett, Lynn Dombrowski, and Gillian R. Hayes. 2016. "Will I always be not social?": Re-Conceptualizing Sociality in the Context of a Minecraft Community for Autism. In Proceedings of the 2016 CHI Conference on Human Factors in Computing Systems (CHI '16). ACM, New York, NY, USA, 1256-1269.

[26] Ashley E. Robertson and David R. Simmons. 2013. The Relationship between Sensory Sensitivity and Autistic Traits in the General Population Journal of Autism and Developmental Disorder, 43: 775. https://doi.org/10.1007/s10803-012-1608-7

[27] Christian Samsel, Karl-Heinz Krempels, and Gerrit Garbereder. 2016. Personalized, Context-aware Intermodal Travel Information. In Proceedings of the 12th International Conference on Web Information Systems and Technologies, WEBIST 2016, Rome, Italy, April 23-25, 2016, Vol. 2. SCITEPRESS, 148-155. DOI: http://dx.doi.org/10.5220/0005855501480155

[28] J. Ben Schafer, Dan Frankowski, Jon Herlocker and Shilad Sen. 2003. Collaborative Filtering Recommender Systems, Springer.

[29] Will Simm, Maria Angela Ferrario, Adrian Gradinar, Marcia Tavares Smith, Stephen Forshaw, Ian Smith, and Jon Whittle. 2016. Anxiety and Autism Towards Personalized Digital Health. In Proceedings of the $2016 \mathrm{CHI}$ Conference on Human Factors in Computing Systems (CHI '16). ACM, New York, NY, USA, 1270-1281. DOI: https://doi.org/10.1145/2858036.2858259

[30] Gytis Tumas and Francesco Ricci. 2009. Personalized Mobile City Transport Advisory System. In Information and Communication Technologies in Tourism 2009: Proceedings of the International Conference in Amsterdam, .e Netherlands, 2009, Wolfram H“opken, Ulrike Gretzel, and Rob Law (Eds.). Springer Vienna,Vienna, 173-183. DOI:h.p://dx.doi.org/10.1007/978-3-21193971-0 15

[31] Catalin Voss, Nick Haber, Peter Washington, Aaron Kline, Beth McCarthy, Jena Daniels, Azar Fazel, Titas De, Carl Feinstein, Terry Winograd, Dennis Wall. 2016. Designing a Holistic At-Home Learning Aid for Autism. CHI'16 Workshop Proceedings.

[32] Thorsten Völkel and Gerhard Weber. 2008. RouteCheckr: personalized multicriteria routing for mobility impaired pedestrians. In Proceedings of the 10th international ACM SIGACCESS conference on Computers and accessibility (Assets '08). ACM, New York, NY, USA, 185-192. 